\title{
A study of Sirt1 regulation and the effect of resveratrol on synoviocyte invasion and associated joint destruction in rheumatoid arthritis
}

\author{
LIANG HAO ${ }^{1 *}$, YUYING WAN $^{2 *}$, JUHUA XIAO $^{3}$, QIANG TANG $^{1}$, HUAN DENG $^{4}$ and LU CHEN ${ }^{1}$ \\ Departments of ${ }^{1}$ Orthopedics and ${ }^{2}$ Infection Control, The Second Affiliated Hospital of Nanchang University, Nanchang, \\ Jiangxi 300006; ${ }^{3}$ Department of Ultrasound, Jiangxi Provincial Maternal and Child Health Hospital; ${ }^{4}$ Department of \\ Pathology, The Fourth Affiliated Hospital of Nanchang University, Nanchang, Jiangxi 330006, P.R. China
}

Received March 27, 2016; Accepted March 17, 2017

DOI: $10.3892 / \mathrm{mmr} .2017 .7299$

\begin{abstract}
The aim of the current study was to investigate the role and mechanism of sirtuin 1 (Sirt1) in the regulation of synovial cell invasion and joint destruction in rheumatoid arthritis (RA). The Sirt1 protein and mRNA levels in fibroblast-like synoviocytes (FLS) isolated from RA synovial tissues were compared with normal tissues by western blot and reverse transcription-polymerase chain reaction. RA FLS were then treated with the Sirt1 agonist resveratrol $(1,3$ and $10 \mu \mathrm{g} / \mathrm{ml})$ for $48 \mathrm{~h}$, and their invasiveness and expression of matrix metalloproteinase (MMP) 1 and MMP13 protein and mRNA were measured. Furthermore, a collagen-induced arthritis (CIA) rat model was established and the rats were divided into a model group, and low- and high-dose resveratrol ( 2.5 and $10 \mathrm{mg} / \mathrm{kg} / \mathrm{day}$ ) groups to receive an intraperitoneal injection of resveratrol for 42 consecutive days. The joint morphology, arthritis index (AI), and MMP1 and MMP13 expression in synovial tissues was monitored. The Sirt1 protein and mRNA levels in RA FLS were significantly lower compared with normal FLS $(\mathrm{P}<0.01)$. The resveratrol treatment significantly inhibited the invasive ability of RA FLS $(\mathrm{P}<0.01)$ and reduced MMP1 and MMP13 expression $(\mathrm{P}<0.01)$. The $\mathrm{AI}$ in low- and high-dose groups was significantly lower compared with the model group from day 28 $(\mathrm{P}<0.01)$. Resveratrol also reduced the swelling and damage and decreased MMP1 and MMP13 expression levels in CIA rats $(\mathrm{P}<0.01)$. The resveratrol-induced upregulation of Sirt1 in RA FLS may significantly inhibit the invasion of these cells and reduce the degree of joint damage, which may be mediated
\end{abstract}

Correspondence to: Dr Lu Chen, Department of Orthopedics, The Second Affiliated Hospital of Nanchang University, 1 Minde Road, East Lake, Nanchang, Jiangxi 300006, P.R. China

E-mail: trhf65093615@126.com

*Contributed equally

Key words: rheumatoid arthritis, silent information regulation of transcription 1, fibroblast-like synoviocytes, invasion, joint destruction through the inhibition of MMP1 and MMP13 expression. The present results suggested a regulatory role for Sirt1 in RA pathogenesis, and demonstrated the beneficial effects of resveratrol, which may have potential as an alternative therapeutic strategy for the treatment of patients with RA.

\section{Introduction}

Rheumatoid arthritis (RA) is a systemic autoimmune disease that affects the peripheral joint symmetry, leading to erosive arthritis $(1,2)$. RA is a widely prevalent disease with a high rate of morbidity and mortality. RA development gives rise to abnormal hyperplasia of synovial cells, forming synovial pannus with inflammatory cells and small blood vessels. Matrix metalloproteinases (MMPs) are secreted by the synovial pannus into synovial fluid and then transferred to the extracellular matrix, leading to degradation of articular bones and cartilages (3-6).

Fibroblast-like synoviocytes (FLS) are the major cells in articular synovial tissues and important components of synovial lining tissues. FLS release multiple cytokines and have an important role in joint destruction. Recently, the invasion of FLS has been recognized as a novel target for RA therapy (3-6). Sirtuin 1 (Sirt1) is one of the most well-studied members of the sirtuin protein family. As a nicotinamide adenine dinucleotide ${ }^{+}$-dependent histone deacetylase present in all mammalian somatic cells, the Sirtl protein is involved in several diseases, including neurodegenerative diseases, diabetes, cancer and inflammation. It has been reported that Sirt1 exhibits abnormal expression in RA synovial tissues and has an important role in the development of the disease (7-9). Furthermore, the therapeutic effects of Sirt1 agonists have been observed in mice with collagen-induced arthritis (CIA) (10-12). Sirt1 is also associated with the invasiveness of a variety of tumor cells $(13,14)$. We therefore hypothesize that Sirt1 may be closely associated with RA FLS invasion and Sirt1 expression level may directly affect the invasiveness of these cells. In this study, the expression level of Sirt1 protein and mRNA in RA FLS was examined by western blot and reverse transcription-polymerase chain reaction (RT-PCR) analyses. Resveratrol has been known as an Sirtl agonist due 
to its ability to enhance the deacetylase activity of Sirt1 (15). The RA FLS were then treated with resveratrol for $48 \mathrm{~h}$, and their invasiveness and MMPs expression levels were measured. Further, a CIA rat model was established to evaluate effect of Sirtl activation by resveratrol on the arthritis index (AI), joint morphology and MMP expression levels in these rats.

\section{Materials and methods}

Reagents and instruments. Reagents in this study included resveratrol and collagen II (Sigma-Aldrich; Merck KGaA, Darmstadt, Germany), Dulbecco's modified Eagle's medium (DMEM), fetal bovine serum (FBS), penicillin-streptomycin double-resistance (Gibco; Thermo Fisher Scientific, Inc., Waltham, MA, USA), tetrazolium reagent (MTT; Sigma-Aldrich; Merck KGaA), rabbit anti-human MMP1 (cat no. 1973-1) and rabbit anti-human MMP13 (cat no. 1923-1) monoclonal antibodies (Epitomics, Burlingame, CA, USA), rabbit anti-human Sirt1 polyclonal antibody (cat no. ab110304; Abcam, Cambridge, MA, USA), protein extraction kit, bicinchoninic acid (BCA) protein assay kit, mouse anti-human GAPDH monoclonal antibody (cat no. AG019), horseradish peroxidase (HRP)-labeled goat anti-rabbit immunoglobulin (Ig) G (cat no. A0208), HRP-labeled goat anti-mouse IgG (cat no. A0216), ECL supersensitive luminescent liquid (Beyotime Institute of Biotechnology, Haimen, China), TRIzol reagent and one-step RT-PCR kit (Invitrogen; Thermo Fisher Scientific, Inc.). Transwell chambers (Corning Incorporated, Corning, NY, USA), ChemiDocTM XRS gel imaging system (Bio-Rad Laboratories, Inc., Hercules, CA, USA) and TE2000 inverted fluorescence microscope (Nikon Corporation, Tokyo, Japan) were used.

Synovial tissues. Rheumatoid synovial tissues were collected from 20 patients with rheumatoid disease that underwent knee replacement surgery in the Department of Orthopedics, The Second Affiliated Hospital of Nanchang University (Nanchang, China) between December 2013 and December 2015. All patients met the classification standard of American Rheumatology Society (16). Normal synovial tissues were obtained from non-rheumatoid patients that had undergone orthopedic amputations due to accidental injury. The study was approved by the Ethics Committee of The Second Affiliated Hospital of Nanchang University. All patients were required to provide signed informed consent.

\section{In vitro cell experiments}

FLS isolation and culture. The collected synovial tissues were washed three times with PBS under sterile conditions. After removing fat with a blade, the tissues were cut into small pieces with surgical scissors and digested with $0.25 \%$ trypsin and $3 \%$ collagenase for $30 \mathrm{~min}-3 \mathrm{~h}$ to obtain single cell suspensions. The cells were re-suspended in DMEM medium (10\% FBS, $100 \mathrm{U} / \mathrm{ml}$ penicillin-streptomycin) and incubated at $37^{\circ} \mathrm{C}$ in an incubator with $5 \% \mathrm{CO}_{2}$. Cell fusion occurred after 5 or 6 days, and cells at passage 2-3 were used for subsequent experiments.

Cell invasion assay. The RA FLS in the logarithmic growth phase $\left(1 \times 10^{4}\right.$ cells) were seeded on 6 -well plates. After $24 \mathrm{~h}$, they were treated with 1,3 , and $10 \mu \mathrm{g} / \mathrm{ml}$ resveratrol, and untreated RA FLS were used as the control. After $48 \mathrm{~h}$, the cells were trypsinized and added to the upper chamber of a Transwell device in serum-free DMEM, and the lower chamber was filled with 5\% DMEM containing 10\% FBS. The device was incubated for another $24 \mathrm{~h}$. The lower chamber was removed, washed, fixed with $0.4 \%$ paraformaldehyde at room temperature for $5 \mathrm{~min}$, and stained with crystal violet. Cell invasion was measured by counting the number of invaded cells under an inverted optical microscope. Mean values were obtained from five randomly selected visual fields for each well.

Cell viability assay. RA FLS in the logarithmic growth phase were seeded in 96-well plates at a density of $6 \times 10^{3}$ cells/well. After $24 \mathrm{~h}$, they were treated with 1,3 , and $10 \mu \mathrm{g} / \mathrm{ml}$ resveratrol, and untreated RA FLS were used as the control group. After 48 h, $20 \mu 15 \mathrm{mg} / \mathrm{ml}$ MTT was added, followed by an addition of $150 \mu \mathrm{l}$ of DMSO after $4 \mathrm{~h}$. The optical density value representing the cell viability was then measured with a microplate reader (Bio-Rad Laboratories, Inc.) at $560 \mathrm{~nm}$.

\section{In vivo animal experiments}

Modeling and grouping. A total of 504 Sprague-Dawley (SD) rats (age, 4 weeks; weight, $200 \pm 20 \mathrm{~g}$ ) were purchased from Slack Animal Experiments, LLC [Shanghai, China; certificate: SCXK (Shanghai) 2012-0002]. The rats were housed at $23 \pm 2{ }^{\circ} \mathrm{C}$ under a 12 -h light/dark cycle with free access to food and water. The CIA rat model was established as previously described (17). Briefly, the rats were given a subcutaneous injection of $100 \mu 12 \mathrm{~g} / \mathrm{l}$ type II collagen emulsion in the right-back toe pad on day 1 and a booster injection of the same dose on day 7 . The normal group received an equal volume of saline at the same time points. The CIA rats were randomly divided into model group, low-dose resveratrol group, and high-dose resveratrol group (n=10 rats/group). The low-dose and high-dose groups were intraperitoneally injected with 2.5 and $10 \mathrm{mg} / \mathrm{kg}$ resveratrol per day for 42 consecutive days, respectively. The normal and model groups were injected with an equal amount of saline. The animal study was approved by the Ethics Committee of the Second Affiliated Hospital of Nanchang University.

AI assessment. The AI of each rat was assessed as previously described (18). Briefly, the hind limb and joint were observed at intervals of 7 days after the first day of the model. The joints were evaluated with a scale of $0-4$, for which 0 indicates normal joint, 1 for mild joint swelling, 2 for moderate swelling, 3 for severe joint swelling incapable of bending and walking, and 4 for severe swelling incapable of weight-loading.

Joint X-ray assessment. All rats were subjected to joint X-ray examination at day 42 (19). Each rat was anesthetized by an intraperitoneal injection of $70 \mathrm{mg} / \mathrm{kg}$ of $0.6 \%$ sodium pentobarbital, and the right-back toe was examined for joint destruction using a dental X-ray machine (with a setup of $1 \mathrm{sec}, 60 \mathrm{KV}, 3 \mathrm{~mA})$.

Collection of synovial tissues. At day 42, rats were sacrificed. The synovial tissues were collected, homogenized with a homogenizer and subjected to subsequent experiments. 


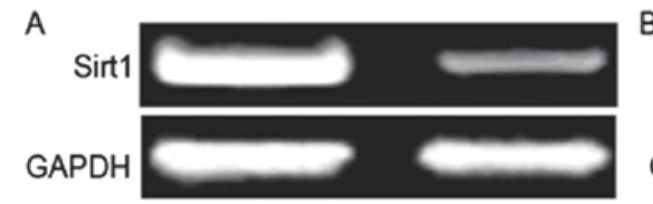

Normal

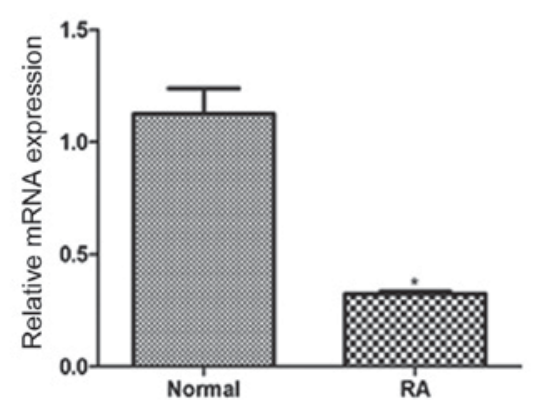

RA
B
SArt1
GAPDH

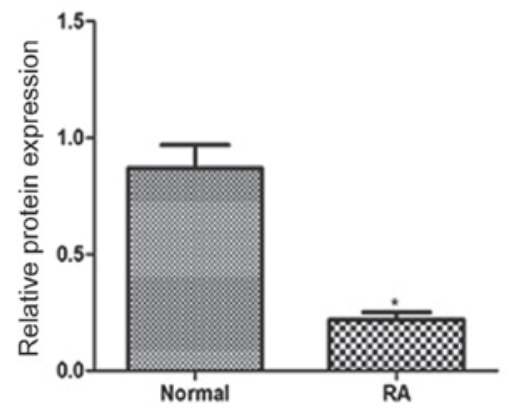

Figure 1. Comparison of Sirt1 mRNA and protein expression in normal fibroblast-like synoviocytes and from patients with RA. (A) Reverse transcription-polymerase chain reaction and (B) western blot analyses. ${ }^{*} \mathrm{P}<0.01$. Sirt1, sirtuin 1 ; RA, rheumatoid arthritis.

Western blot analysis. The expression levels of MMP1, MMP13 and Sirt1 in RA FLS and synovial tissues in CIA rats were determined by western blot analyses. Briefly, total protein was extracted using protein extraction kits and quantified using a BCA kit according to the manufacturer's instruction. Equal amounts of total protein $(10 \mu \mathrm{g})$ were separated by $12 \%$ SDS-PAGE and transferred to polyvinylidene difluoride membranes at $100 \mathrm{~V}$ for $1 \mathrm{~h}$. The membrane was blocked in TBS buffer containing 5\% skim milk and $0.1 \%$ Tween-20 at room temperature for $1 \mathrm{~h}$, and incubated with the following primary antibodies overnight at $4^{\circ} \mathrm{C}$ : Anti-MMP-1, anti-MMP-13, anti-sirt1 (1:100) and anti-GAPDH (1:200). The membrane was washed three times with TBST for $5 \mathrm{~min}$ each, and incubated with HRP-labeled goat anti-rabbit or goat anti-mouse $\operatorname{IgG}(1: 500)$ at room temperature for $1 \mathrm{~h}$. The membranes were washed three times with TBST and subjected to enhanced chemiluminescent detection. The intensity of bands was detected by a Molecular Imager ${ }^{\circledR}$ ChemiDocTM XRS System (Bio-Rad Laboratories, Inc.). The gray value of bands was analyzed by Image Lab 2.0 software (Bio-Rad Laboratories, Inc.). The relative expression level of target protein was calculated as the ratio of gray value of target protein to that of the internal control (GAPDH). The experiments were performed in triplicate.

RT-PCR analysis. The mRNA levels of MMP1, MMP13 and Sirt1 in synovial tissue and synovial cells were detected by RT-PCR analyses. Total RNA in RA FLS or synovial tissue was extracted from with TRIzol reagent (Invitrogen, Shanghai, China) according to the manufacturer's instructions. RNA concentration was determined using a spectrometer under a wavelength of $260 \mathrm{~nm}$. The total RNAs were reverse transcribed into cDNA using the HiFi Script cDNA kit (CWBio, Beijing, China) according to the manufacture's protocol. The temperature protocol that was used was as follows: at $50^{\circ} \mathrm{C}$ for $1 \mathrm{~h}$ and at $80^{\circ} \mathrm{C}$ for $5 \mathrm{~min}$. qPCR amplification was performed on cDNA using Premix Ex Taq (Takara Biotechnology Co., Ltd., Dalian, China). The primer sequences were as follows: Sirt1, forward 5'-TGGACTCCA
CGACGTACT, reverse 5'-TCTCCTGGGAGGCATAGACC; MMP1, forward 5'-ACTCTGGAGTAATGTCACACCT, reverse 5'-GTTGGTCCACCTTTCATCTTCA; MMP13, forward 5'-ATGGATGTCTTCAAGAAGGG, reverse 5'-CTA GTCTCCCCCACTCTGGG; GAPDH, forward 5'-AGCCAC ATCGCTCAGACA, reverse 5'-TCTCCTGGGAGGCAT AGACC. The PCR program was as follows: $95^{\circ} \mathrm{C}$ for $4 \mathrm{~min}$, followed by 40 cycles of $95^{\circ} \mathrm{C}$ for $40 \mathrm{sec}, 58^{\circ} \mathrm{C}$ for $30 \mathrm{sec}$, and $72^{\circ} \mathrm{C}$ for $30 \mathrm{sec}$. The PCR products $(5 \mu \mathrm{l})$ were examined on $2 \%$ agarose gel, and the nucleotide bands were detected with a gel imaging system (Bio-Rad Laboratories, Inc.). The DNA marker (D0107) was purchased from Beyotime Institute of Biotechnology.

Statistical analysis. Three replicates were performed for each test and all data were expressed as the mean \pm standard deviation. Statistical analyses were performed using SPSS 17.0 (SPSS Inc., Chicago, IL, USA). Differences among groups were compared by one-way analysis of variance, followed by least significant difference test. $\mathrm{P}<0.05$ was considered to indicate a statistically significant difference.

\section{Results}

Sirtl expression in RA FLS. As presented in Fig. 1, the relative Sirt1 mRNA expression level in RA FLS $(0.32 \pm 0.03)$ was significantly lower than that in normal FLS $(1.13 \pm 0.14$, $\mathrm{P}<0.01)$. Similarly, the relative Sirt1 protein level in RA FLS $(0.22 \pm 0.03)$ was also significantly lower compared with normal FLS $(0.87 \pm 0.11, \mathrm{P}<0.01)$.

Resveratrol upregulates Sirtl expression. As presented in Fig. 2, treatment of RA FLS with resveratrol $(1,3$ and $10 \mu \mathrm{g} / \mathrm{ml})$ significantly increased the expression level of Sirt1 mRNA and protein compared with the control group $(\mathrm{P}<0.01)$. The highest Sirt1 expression was observed in the $10 \mu \mathrm{g} / \mathrm{ml}$ resveratrol group, followed successively by the 3 and $1 \mu \mathrm{g} / \mathrm{ml}$ groups, suggesting a dose-dependent stimulatory effect of resveratrol on Sirt1 expression. 

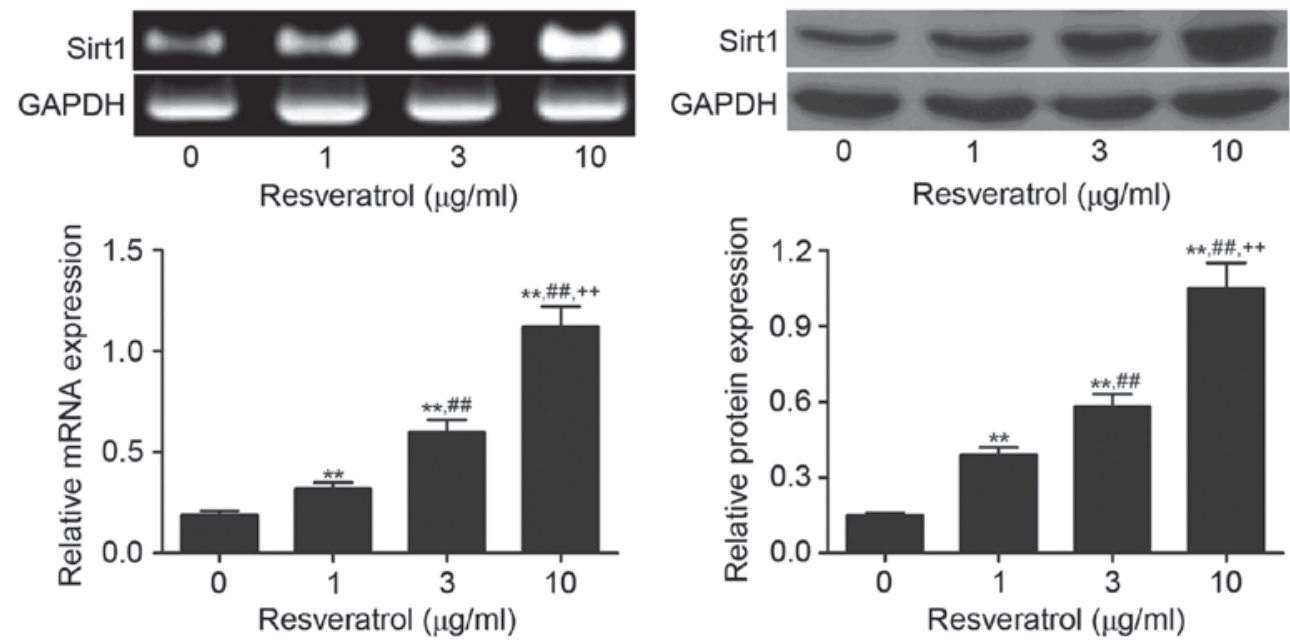

Figure 2. Detection of Sirt1 protein and mRNA expression in rheumatoid arthritis fibroblast-like synoviocytes treated with 1,3 and $10 \mu \mathrm{g} / \mathrm{ml}$ resveratrol by reverse transcription-polymerase chain reaction and western blot analyses. ${ }^{* *} \mathrm{P}<0.01 \mathrm{vs} .0 \mu \mathrm{g} / \mathrm{ml}$ control group; ${ }^{\# \#} \mathrm{P}<0.01 \mathrm{vs} .1 \mu \mathrm{g} / \mathrm{ml}$ resveratrol group; ${ }^{++} \mathrm{P}<0.01$ vs. $3 \mu \mathrm{g} / \mathrm{ml}$ resveratrol group. Sirt1, sirtuin 1 .

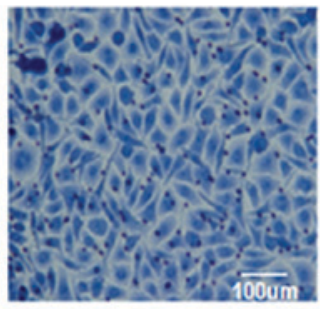

A

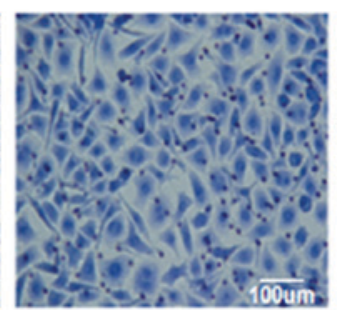

B

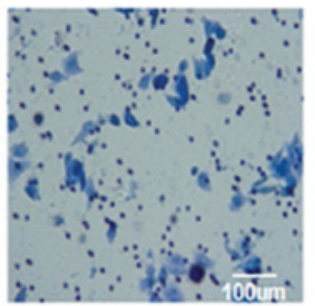

C

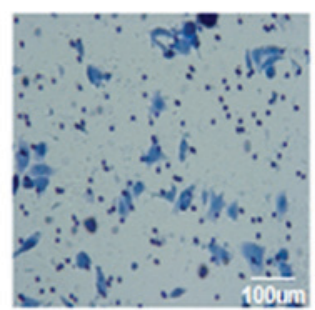

D

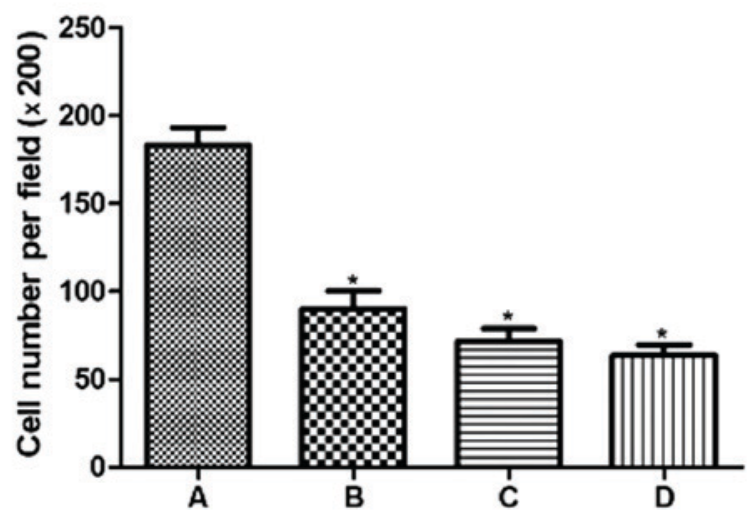

Figure 3. Effect of resveratrol on rheumatoid arthritis fibroblast-like synoviocyte invasiveness. Crystal violet staining of invaded cells in a Transwell assay from (A) control cells without resveratrol treatment, (B) $1 \mu \mathrm{g} / \mathrm{ml}$ resveratrol, (C) $3 \mu \mathrm{g} / \mathrm{ml}$ resveratrol and (D) $10 \mu \mathrm{g} / \mathrm{ml} \mathrm{resveratrol} \mathrm{groups.} \mathrm{"P}<0.01$. Scale bar, $100 \mu \mathrm{m}$.

Effect of Sirt1 upregulation on RA FLS invasiveness. RA FLS cell invasiveness in different groups was compared by a cell invasion assay. As demonstrated in Fig. 3, the number of invading cells in the treated groups $(90.3 \pm 10.0,71.2 \pm 7.7$, and $63.3 \pm 6.4$ for the treatment of 1,3 , and $10 \mu \mathrm{g} / \mathrm{ml}$ resveratrol groups, respectively) was significantly lower compared with the sham control group $(183.4 \pm 10.2, \mathrm{P}<0.01)$. A significant difference in the number of invasive cells was detected among the three resveratrol groups $(\mathrm{P}<0.01)$, suggesting that the induction of Sirt1 by resveratrol may significantly inhibit the invasion ability of RA FLS cells in a dose-dependent manner. Furthermore, RA FLS cell viability was detected by MTT assay. There was no significant difference in the cell viability among different groups, indicating that resveratrol did not exert a significant impact on the viability of RA FLS cells ( $P>0.05$; Fig. 4).

Effect of the Sirt1 upregulation on the expression level of MMPs in RA FLS. The expression level of MMP1 and MMP13 protein was detected by western blot analyses. As demonstrated in Fig. 5, the MMP1 and MMP13 protein levels in RA FLS treated with resveratrol $(1,3$ or $10 \mu \mathrm{g} / \mathrm{ml})$ was significantly reduced compared to the control group $(\mathrm{P}<0.01)$. Consistently, the resveratrol treatment $(1,3$, or $10 \mu \mathrm{g} / \mathrm{ml})$ also significantly decreased the MMP1 and MMP13 mRNA level in RA FLS ( $<<0.01 ;$ Fig. 6). It was also shown that the MMP1 


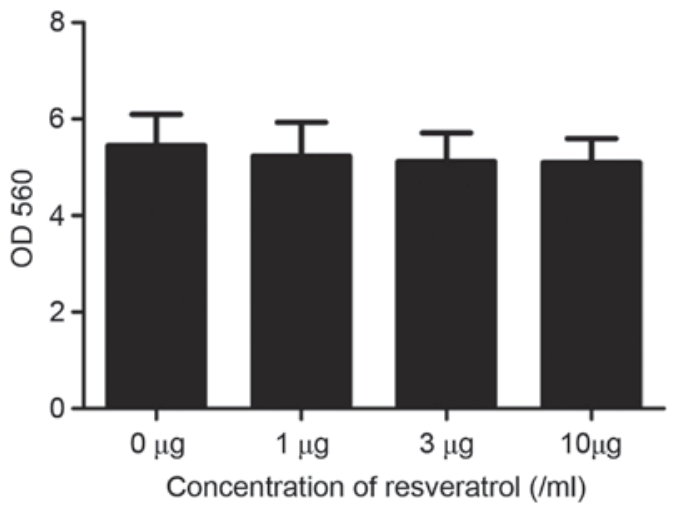

Figure 4. MTT assay comparing the effect of resveratrol on rheumatoid arthritis fibroblast-like synoviocyte cell viability.
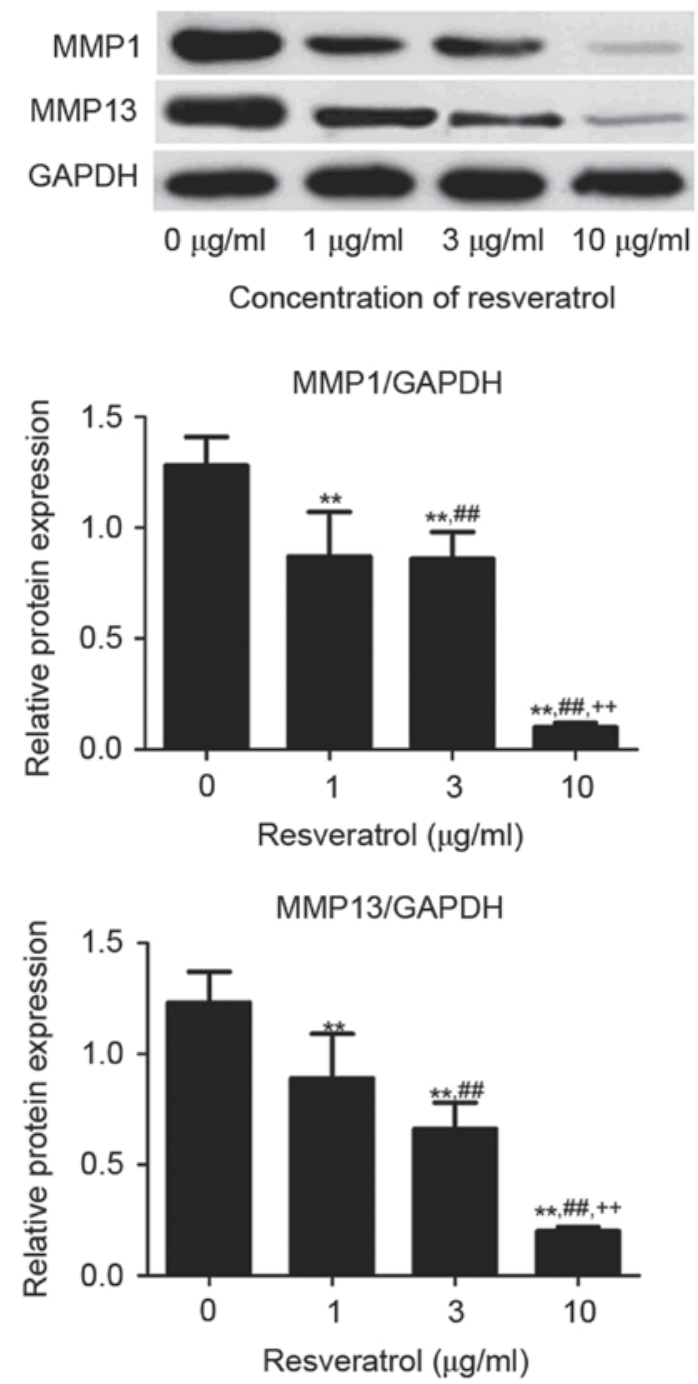

Figure 5. Western blot analyses comparing the expression of MMP proteins in rheumatoid arthritis fibroblast-like synoviocytes treated with resveratrol. ${ }^{* *} \mathrm{P}<0.01$ vs. $0 \mu \mathrm{g} / \mathrm{ml}$ group; ${ }^{\# \#} \mathrm{P}<0.01$ vs. $1 \mu \mathrm{g} / \mathrm{ml}$ resveratrol group; ${ }^{++} \mathrm{P}<0.01$ vs. $3 \mu \mathrm{g} / \mathrm{ml}$ resveratrol group. MMP, matrix metalloproteinase.

and MMP13 protein and mRNA level in $1 \mu \mathrm{g} / \mathrm{ml}$ resveratrol group was the highest, followed successively by 3 and $10 \mu \mathrm{g} / \mathrm{ml}$ groups, indicating that resveratrol dose-dependently inhibited the MMP1 and MMP13 protein and mRNA expression, suggesting that Sirtl upregulation may reduce the expression of these MMPs.

Effect of resveratrol on AI in CIA rats. Of the 50 rats in the study, 40 rats exhibited a marked loss of appetite, activity and hair gloss compared to the normal healthy group, thus producing an $80 \%$ success rate in establishing the CIA model. Furthermore, 30 CIA model rats were randomly selected and grouped into model group, low-dose resveratrol group and high-dose resveratrol group. Sirt1 expression in the synovial tissue of CIA rats was analyzed by western blotting, as shown in Fig. 7: Resveratrol at low and high doses significantly upregulated Sirtl expression in the synovial tissue of CIA rats $(\mathrm{P}<0.01)$. Furthermore, Sirt1 expression in the high-dose group was significantly higher compared with in the low-dose group $(\mathrm{P}<0.01)$. The rat AI was monitored throughout the treatment. As presented in Fig. 8, the AI in the model group began to increase after the first day of modeling, reached the highest level at day 21, and subsequently plateaued ( $\mathrm{P}<0.01$, vs. normal group). The AI in both the low- and high-dose groups began to decrease compared with the model group after day 21 , and reached the lowest levels at day 42. The AI in the low- and high-dose groups were significantly reduced compared with the model group at day 28, 35 and $42(\mathrm{P}<0.01)$, and also the AI in high-dose group was significant lower compared with the low-dose group at these time points $(\mathrm{P}<0.01)$

Effect of the resveratrol on joint morphology in CIA rats. The right-back toe of each rat was examined for joint destruction by X-ray at day 42 of the model. In the normal control group there was normal bone tissue, clearly visible space between the toes, a sharp bone edge and uniform bone mineral density. By contrast, swollen bone tissue, narrowed joint space, blurry bone edge and uneven bone density were observed in the model group. Markedly reduced bone tissue swelling and improved joint space were observed in the high and low dose resveratrol groups compared with the model group, suggesting that Sirt1 upregulation by resveratrol may reduce joint destruction in CIA rats.

Effect of resveratrol on MMP expression levels in the synovial tissue of CIA rats. The expression level of MMP1 and MMP13 protein and mRNA in the synovial tissue of CIA rats was detected by western blot analyses and RT-PCR. As presented in Fig. 9, the MMP1 and MMP13 protein expression in the model group was significantly higher than in the normal group $(\mathrm{P}<0.01)$. Both the high and low dose of resveratrol significantly reduced the level of MMP proteins compared with the model group $(\mathrm{P}<0.01)$. Consistently, the resveratrol treatment at either low or high dose significantly reduced the expression level of MMP1 and MMP13 mRNAs in the synovial tissue in the CIA rats as compared to the model group ( $\mathrm{P}<0.01$; Fig. 10). A dose-dependent inhibitory effect of resveratrol on the MMP1/13 protein and mRNA expression was also observed, indicating that the resveratrol-induced Sirt1 upregulation may mediate the reduced expression of these MMPs. The expression of MMP1 and MMP13 mRNA and protein in high-dose group was significantly reduced compared with low-dose group $(\mathrm{P}<0.01)$. 

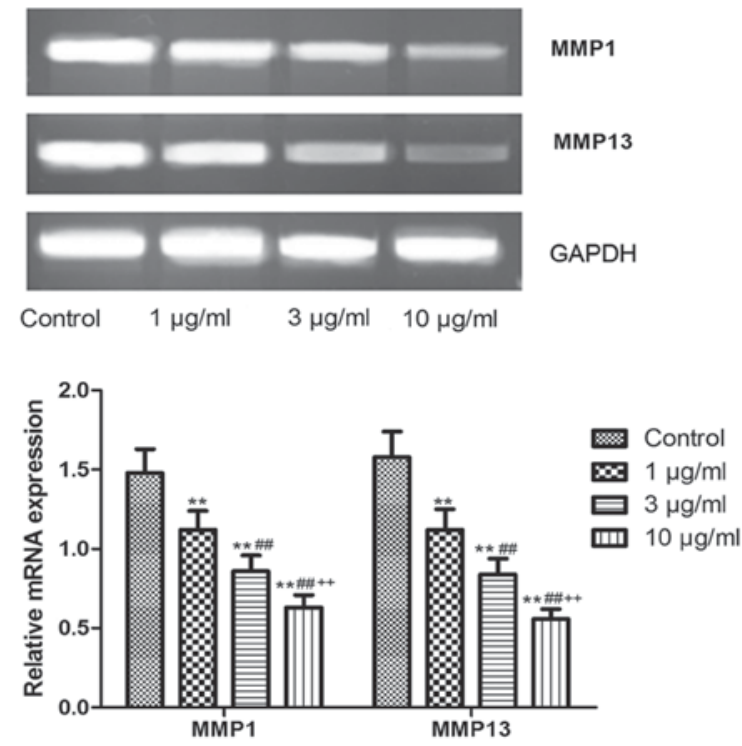

Figure 6. Polymerase chain reaction detected the mRNA expression of MMPs in rheumatoid arthritis fibroblast-like synoviocytes treated with resveratrol. ${ }^{* *} \mathrm{P}<0.01 \mathrm{vs} .0 \mu \mathrm{g} / \mathrm{ml}$ group; ${ }^{* \#} \mathrm{P}<0.01 \mathrm{vs} .1 \mu \mathrm{g} / \mathrm{ml}$ resveratrol group; ${ }^{++} \mathrm{P}<0.01$ vs. $3 \mu \mathrm{g} / \mathrm{ml}$ resveratrol group. MMP, matrix metalloproteinase.

\section{Discussion}

The silence information regulator 2 (Sir2) gene family of deacetylases in yeast chromatin broadly participate in several biological processes, including cell survival, apoptosis and senescence. As a homolog of Sir2, Sirt1 also has deacetylase activity, and interacts with a variety of histone and non-histone proteins to maintain a low level of acetylation in normal cells, and normal expression level of non-histone proteins. Sirt1 is closely associated with a variety of physiological and pathological processes, including cell apoptosis, cell cycle, cell energy metabolism, lipid accumulation and cellular senescence (20). Although several studies have detected abnormal expression of Sirt1 in RA patients, the role of Sirt1 expression in RA has remained controversial. For instance, Wendling et al (8) reported that the Sirt1 protein level in peripheral blood mononuclear cells from RA patients is lower that the levels in healthy people. Wang et al (21) reported that induction of the Sirtl level via inhibition of nuclear factor- $\mathrm{B}(\mathrm{NF}-\kappa \mathrm{B}) \mathrm{p} 65$ protein expression improves arthritis in CIA rats. By contrast, Niederer et al (9) reported that Sirt1 was overexpressed in RA synovial tissue compared with OA. Silencing of the sirtl gene promotes apoptosis of RA FLS, whereas overexpression of this sirtl can represses cell apoptosis. In the current study, the Sirt1 mRNA and protein level in RA FLS was examined by western blot and RT-PCR analyses. The results demonstrated a significantly lower level of Sirt1 expression in RA FLS than in normal control FLS, which is consistent with the study by Wendling et al (8). The current study also revealed that resveratrol dose-dependently increased the level of Sirt1 protein and mRNA in RA FLS.

As a main component of synovial cells, FLS are located in the synovial lining layer and possess 'tumor cell-like' invasive biological behavior. A previous study suggested that RA joints secrete a large amount of inflammatory cytokines and MMPs into the extracellular matrix under low oxygen conditions,
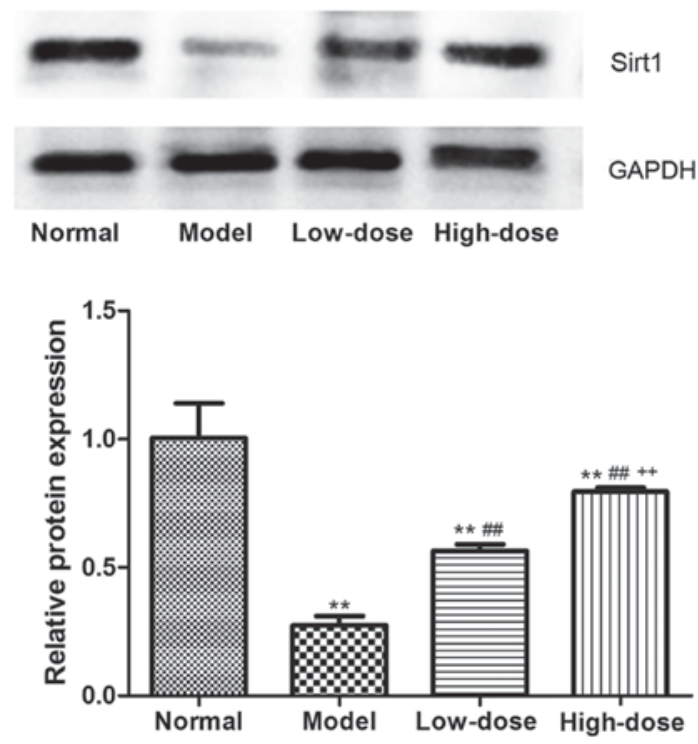

Figure 7. Effect of resveratrol on Sirt1 expression in synovial tissue of collagen-induced arthritis rats. ${ }^{* *} \mathrm{P}<0.01$ vs. normal group; ${ }^{\#} \mathrm{P}<0.01$ vs. model group; ${ }^{++} \mathrm{P}<0.01$ vs. low-dose group. Sirt1, sirtuin 1.

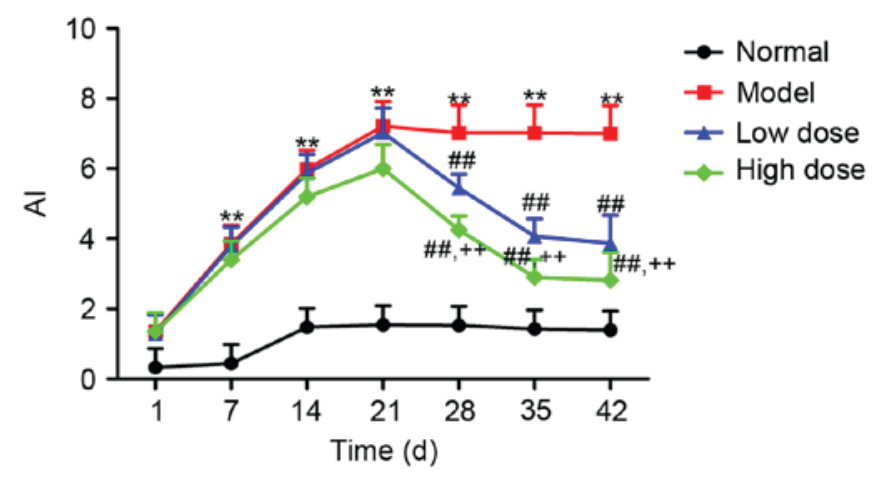

Figure 8. Effect of resveratrol on the AI in collagen-induced arthritis rats ${ }^{* *} \mathrm{P}<0.01$ vs. normal group; ${ }^{\# \#} \mathrm{P}<0.01$ vs. model group; ${ }^{++} \mathrm{P}<0.01$ vs. low-dose group. AI, arthritis index.

leading to the destruction of articular cartilage, bone and joints (22). Lefevre et al (23) reported that RA FLS migrate within intra-articular regions and also across the vascular endothelium, causing degradation of articular cartilage. Videographic examination demonstrated that joints in RA patients with more aggressive FLS are more vulnerable to damage and have higher sharp scores compared with patients with mild or medium FLS (24). Therefore, inhibition of the migration and invasion capacity of RA FLS is expected to become a novel target for the treatment of RA. In this study, resveratrol dose-dependently inhibited the invasion of RA FLS although it did not affect the viability of these cells, indicating that Sirt1 upregulation by resveratrol may markedly reduce the invasive capacity of RA FLS. Previous studies have suggested that resveratrol may exert anti-inflammatory and therapeutic effects in RA through the modulation of phosphatidylinositol-4,5-bisphosphate 3-kinase/Akt pathway (25) and acetylation of activator protein-1 and nuclear factor (NF)- $\mathrm{kB}(26)$.

MMPs, a class of zinc-dependent proteolytic enzymes that are secreted by RA FLS, degrade extracellular matrix, bone 

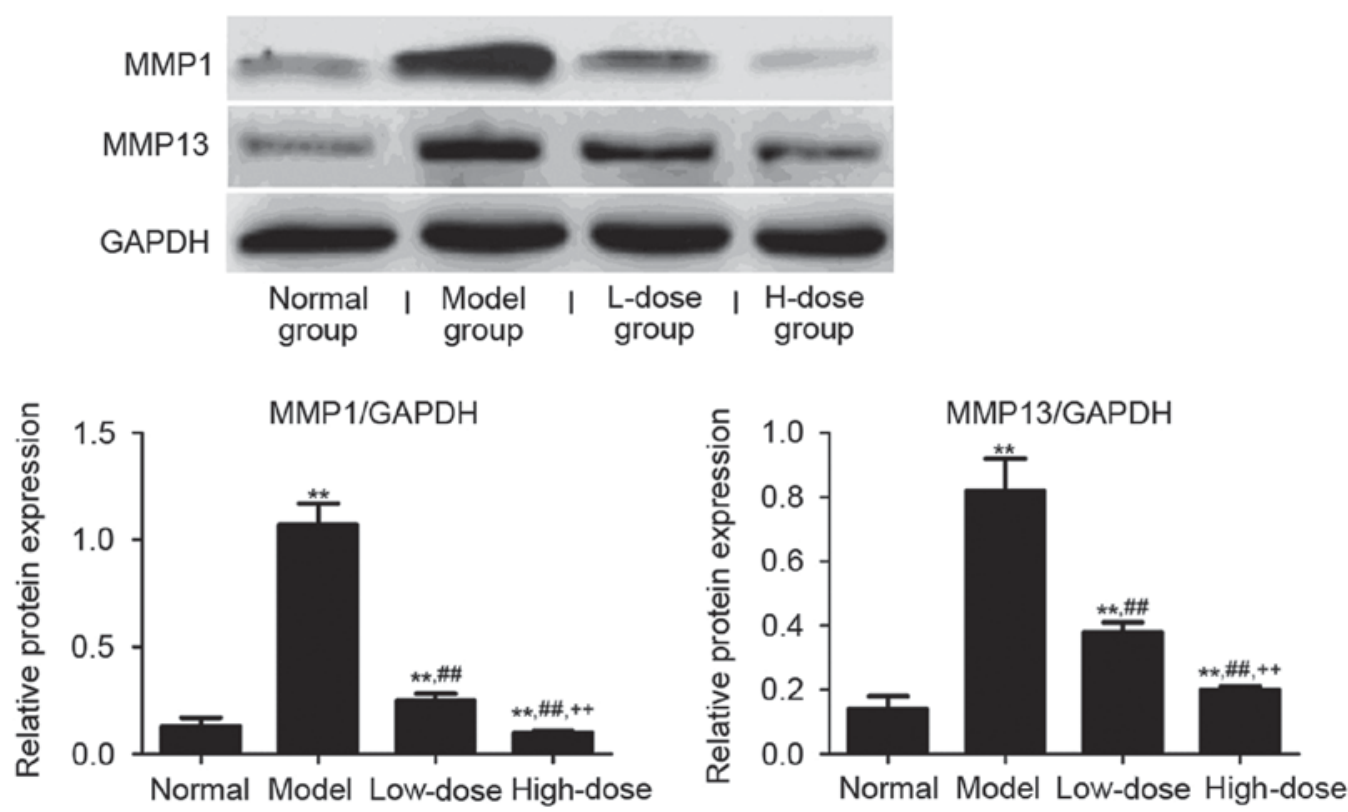

Figure 9. Effect of resveratrol on MMPs expression in synovial tissue of collagen-induced arthritis rats. ${ }^{* *} \mathrm{P}<0.01$ vs. normal group; ${ }^{\# \#} \mathrm{P}<0.01$ vs. model group; ${ }^{++} \mathrm{P}<0.01$ vs. low-dose group. MMP, matrix metalloproteinase.
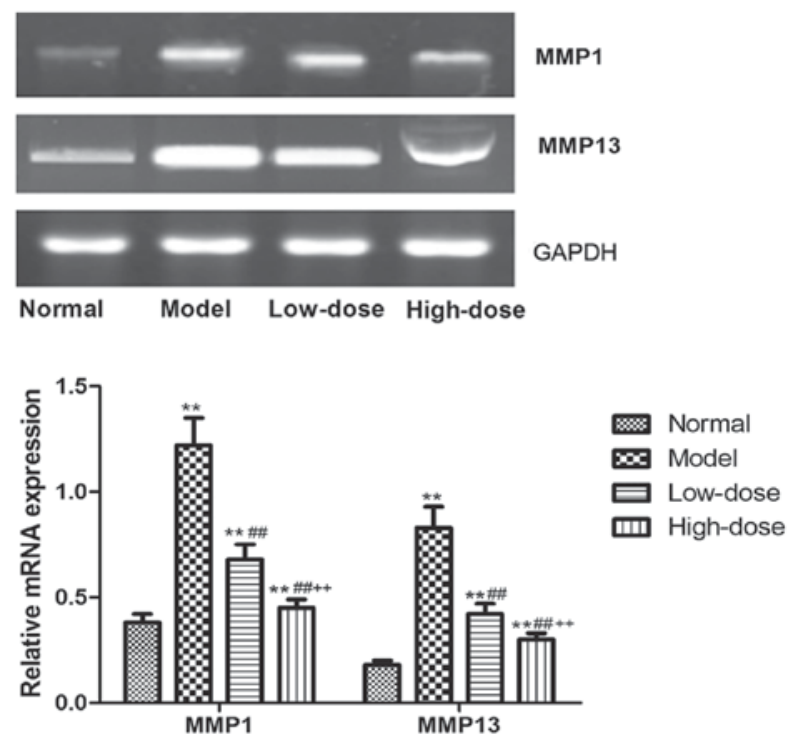

Figure 10. Effect of resveratrol on the mRNA expression levels of MMPs in synovial tissue of collagen-induced arthritis rats. ${ }^{* *} \mathrm{P}<0.01$ vs. normal group; ${ }^{\# \#} \mathrm{P}<0.01$ vs. model group; ${ }^{++} \mathrm{P}<0.01$ vs. low-dose group. MMP, matrix metalloproteinase.

and cartilage, and thus, have an important role in the joint destruction that occurs during RA. High expression levels of MMPs, including MMP1, MMP2 and MMP9, are detected in RA synovial fluid and tissue (27). Aggressive expression of MMP3 and MMP13 was also detected in RA-FLS isolated from patients with RA (28). Furthermore, the migration and invasion ability of RA FLS are significantly inhibited through the downregulation of MMP1 and MMP13 expression $(29,30)$. The current study demonstrated that MMP1 and MMP13 expression in RA FLS was reduced after the induction of Sirt1 by resveratrol at different doses, suggesting that Sirt1 may influence the invasiveness of RA FLS through the regulation of MMP1/13 expression. Nevertheless, further knockdown and overexpression experiments are required to confirm this speculation. Additionally, Sirt1 has been previously demonstrated to inactivate the $\mathrm{NF}-\kappa \mathrm{B}$ signaling pathway through deacetylation of the pathway subunit RelA/p65 to affect the expression of downstream target proteins, such as the inflammatory cytokines TNF- $\alpha$, IL- $1 \beta$ and MMPs (31). Therefore, the association between Sirt1 and the $N F-\kappa B$ signaling pathway in RA may be an important future research focus.

A CIA rat model was established to evaluate the effect of resveratrol-induced Sirt1 upregulation in the treatment of RA. Similar to that in a previous report (17), a modeling success rate of $80 \%$ was achieved. The AI value in the model group was gradually increased and peaked at day 21 , when the most severe joint swelling was also observed during X-ray examination. The rats in the treatment groups received low- or high-dose resveratrol for 42 days, and subsequently the AI, joint morphology, and MMP expression levels in synovial tissue were monitored. The AI in both the low- and high-dose groups began to decrease after day 21 of the model, and reached the lowest level at day 42 . At day 42 of the model, $\mathrm{X}$-ray examination of joint morphology demonstrated that resveratrol treatment at both doses reduced the joint swelling and bone destruction, and improved joint spaces and the bone edge. These results confirmed that upregulation of Sirt1 by resveratrol may decrease the AI in CIA rats, restore the joint morphology and reduce the joint destruction. Consistently, Kim et al (10) have also reported a therapeutic effect of resveratrol in CIA rats. Furthermore, the MMP1 and MMP13 expression in synovial tissue were substantially reduced by resveratrol treatment, indicating that the therapeutic effect of resveratrol may be associated with the downregulation of MMP protein.

In summary, the in vitro experiments in the present study revealed that Sirt1 expression was lower in RA FLS compared with normal FLS, and an upregulation of Sirt1 expression in RA 
FLS was achieved by treatment with resveratrol. Resveratrol treatment also significantly inhibited the invasiveness of RA FLS, potentially by reducing the expression of MMP1 and MMP13. Further animal studies confirmed that the induction of Sirt1 by resveratrol significantly decreased the AI value, restored joint morphology, reduced joint damage and lowered the MMP1/MMP13 expression in CIA rats, suggesting that Sirt1 may regulate synoviocyte invasion and the associated joint destruction by inhibiting the expression of MMP proteins. The present results suggested a regulatory role for Sirt1 in RA pathogenesis, and demonstrated the beneficial effects of resveratrol, which may have potential as an alternative therapeutic strategy for the treatment of patients with RA.

\section{Acknowledgements}

The present study was supported by the Youth Science Foundation of Jiangxi Province (no. 20161BAB215246).

\section{References}

1. Matcham F, Ali S, Hotopf M and Chalder T: Psychological correlates of fatigue in rheumatoid arthritis: A systematic review. Clin Psychol Rev 39: 16-29, 2015.

2. Chi L, Zhou B, Gao W et al: Research advance on the common drugs for the rheumatoid arthritis treatment. Traditional Chin Med 39: 2851-2858, 2014.

3. Bartok B, Hammaker D and Firestein GS: Phosphoinositide 3 -kinase $\delta$ regulates migration and invasion of synoviocytes in rheumatoid arthritis. J Immunol 192: 2063-2070, 2014.

4. Li D, Xiao Z, Wang G and Song X: Knockdown of ADAM10 inhibits migration and invasion of fibroblast-like synoviocytes in rheumatoid arthritis. Mol Med Rep 12: 5517-5523, 2015.

5. Miller MC, Manning HB, Jain A, Troeberg L, Dudhia J, Essex D, Sandison A, Seiki M, Nanchahal J, Nagase H and Itoh Y: Membrane type 1 matrix metalloproteinase is a crucial promoter of synovial invasion in human rheumatoid arthritis. Arthritis Rheum 60: 686-697, 2009.

6. Marrelli A, Cipriani P, Liakouli V, Carubbi F, Perricone C, Perricone R and Giacomelli R: Angiogenesis in rheumatoid arthritis: A disease specific process or a common response to chronic inflammation? Autoimmun Rev 10: 595-598, 2011.

7. Deng Z, Zhao Y, Chen S, et al: Research progress of Stat3 and SirT1 in the rheumatoid arthritis pathogenesis. J Med Postgraduates 2515: 28: 1001-1003.

8. Wendling D, Abbas W, Godfrin-Valnet M, Kumar A, Guillot X, Khan KA, Vidon C, Coquard L, Toussirot E, Prati C and Herbein G: Dysregulated serum IL-23 and SIRT1 activity in peripheral blood mononuclear cells of patients with rheumatoid arthritis. PLoS One 10: e119981, 2015.

9. Niederer F, Ospelt C, Brentano F, Hottiger MO, Gay RE, Gay S, Detmar M and Kyburz D: SIRT1 overexpression in the rheumatoid arthritis synovium contributes to proinflammatory cytokine production and apoptosis resistance. Ann Rheum Dis 70: 1866-1873, 2011.

10. Kim HY, Park SY, Lee SW, Lee HR, Lee WS, Rhim BY, Hong KW and Kim CD: Inhibition of HMGB1-induced angiogenesis by cilostazol via SIRT1 activation in synovial fibroblasts from rheumatoid arthritis. PLoS One 9: e104743, 2014.

11. Glehr M, Breisach M, Walzer S, Lohberger B, Fürst F, Friesenbichler J, Rinner B, Avian A, Windhager R and Leithner A: The influence of resveratrol on the synovial expression of matrix metalloproteinases and receptor activator of NF-kappaB ligand in rheumatoid arthritis fibroblast-like synoviocytes. Z Naturforsch C 68: 336-342, 2013.

12. Tian J, Chen JW, Gao JS, Li L and Xie X: Resveratrol inhibits TNF- $\alpha$-induced IL-1 $\beta$, MMP-3 production in human rheumatoid arthritis fibroblast-like synoviocytes via modulation of PI3kinase/Akt pathway. Rheumatol Int 33: 1829-1835, 2013.
13. Lovaas JD, Zhu L, Chiao CY, Byles V, Faller DV and Dai Y: SIRT1 enhances matrix metalloproteinase-2 expression and tumor cell invasion in prostate cancer cells. Prostate 73: 522-530, 2013.

14. Chen GQ, Tian H, Yue WM, Li L, Li SH, Qi L, Gao C, Si LB, Lu M and Feng F: SIRT1 expression is associated with lymphangiogenesis, lymphovascular invasion and prognosis in $\mathrm{pNO}$ esophageal squamous cell carcinoma. Cell Biosci 4: 48, 2014.

15. Borra MT, Smith BC and Denu JM: Mechanism of human SIRT1 activation by resveratrol. J Biol Chem 280: 17187-17195, 2005.

16. Aletaha D, Neogi T, Silman AJ, Funovits J, Felson DT, Bingham CO III, Birnbaum NS, Burmester GR, Bykerk VP, Cohen MD, et al: 2010 Rheumatoid arthritis classification criteria: An American College of Rheumatology/European League Against Rheumatism collaborative initiative. Ann Rheum 69: 2569-2581, 2010.

17. Brand DD, Kang AH and Rosloniec EF: The mouse model of collagen-induced arthritis. Methods Mol Med 102: 295-312, 2004.

18. Sumariwalla PF, Cao Y, Wu HL, Feldmann M and Paleolog EM: The angiogenesis inhibitor protease-activated kringles 1-5 reduces the severity of murine collagen-induced arthritis. Arthritis Res Ther 5: R32-R39, 2003.

19. Li GQ, Zhang Y, Liu D, Qian YY, Zhang H, Guo SY, Sunagawa M, Hisamitsu T and Liu YQ: PI3 kinase/Akt/HIF-1 $\alpha$ pathway is associated with hypoxia-induced epithelial-mesenchymal transition in fibroblast-like synoviocytes of rheumatoid arthritis. Mol Cell Biochem 372: 221-231, 2013.

20. Seo JS, Moon MH, Jeong JK, Seol JW, Lee YJ, Park BH and Park SY: SIRT1, a histone deacetylase, regulates prion protein-induced neuronal cell death. Neurobiol Aging 33: 1110-1120, 2012.

21. Wang HZ, Wang HH, Huang SS, Zhao H, Cao YG, Wang GZ, Wang D, Wang ZG and Liu YH: Inhibitory effect of baicalin on collagen-induced arthritis in rats through the nuclear factor- $\kappa \mathrm{B}$ pathway. J Pharmacol Exp Ther 350: 435-443, 2014.

22. Bartok B and Firestein GS: Fibroblast-like synoviocytes: Key effector cells in rheumatoid arthritis. Immunol Rev 233: 233-255, 2010.

23. Lefevre S, Meier FM, Neumann E and Muller-Ladner U: Role of synovial fibroblasts in rheumatoid arthritis. Curr Pharm Des 21: 130-141, 2015.

24. Tolboom TC, van der Helm-Van Mil AH, Nelissen RG, Breedveld FC, Toes RE and Huizinga TW: Invasiveness of fibroblast-like synoviocytes is an individual patient characteristic associated with the rate of joint destruction in patients with rheumatoid arthritis. Arthritis Rheum 52: 1999-2002, 2005.

25. Tian J, Chen JW, Gao JS, Li L and Xie X: Resveratrol inhibits TNF- $\alpha$-induced IL-1 $\beta$, MMP-3 production in human rheumatoid arthritis fibroblast-like synoviocytes via modulation of PI3kinase/Akt pathway. Rheumatol Int 33: 1829-1835, 2013.

26. Yang CM, Chen YW, Chi PL, Lin CC and Hsiao LD: Resveratrol inhibits BK-induced COX-2 transcription by suppressing acetylation of AP-1 and NF- $\mathrm{BB}$ in human rheumatoid arthritis synovial fibroblasts. Biochem Pharmacol 132: 77-91, 2017.

27. Kim KS, Lee YA, Choi HM, Yoo MC and Yang HI: Implication of MMP-9 and urokinase plasminogen activator (uPA) in the activation of pro-matrix metalloproteinase (MMP)-13. Rheumatol Int 32: 3069-3075, 2012.

28. Fan W, Zhou ZY, Huang XF, Bao CD and Du F: Deoxycytidine kinase promotes the migration and invasion of fibroblast-like synoviocytes from rheumatoid arthritis patients. Int J Clin Exp Pathol 6: 2733-2744, 2013.

29. Gao J, Zheng W, Wang L and Song B: A disintegrin and metallproteinase 15 knockout decreases migration of fibroblast-like synoviocytes and inflammation in rheumatoid arthritis. Mol Med Rep 11: 4389-4396, 2015.

30. Jie LG, Huang RY, Sun WF, Wei S, Chu YL, Huang QC and Du HY: Role of cysteinerich angiogenic inducer 61 in fibroblast-like synovial cell proliferation and invasion in rheumatoid arthritis. Mol Med Rep 11: 917-923, 2015.

31. Annabi B, Lord-Dufour S, Vézina A and Béliveau R: Resveratrol Targeting of Carcinogen-Induced Brain Endothelial Cell Inflammation Biomarkers MMP-9 and COX-2 is Sirt1-Independent. Drug Target Insights 6: 1-11, 2012. 